\title{
Riqueza e composição de vertebrados em latrinas ativas e inativas de Pteronura brasiliensis (Carnivora, Mustelidae) na Amazônia Oriental, Brasil
}

\author{
Cintia M. Togura ${ }^{1,2}$, Darren Norris ${ }^{2,3} \&$ Fernanda Michalski ${ }^{2,4}$
}

\begin{abstract}
1. Programa de Pós-Graduação em Biodiversidade Tropical, Universidade Federal do Amapá (UNIFAP), Rod. Juscelino Kubitscheck, Km 02, 68902-280, Macapá, AP, Brasil. (cintia_togura@yahoo.com.br)

2. Laboratório de Ecologia e Conservação de Vertebrados, Universidade Federal do Amapá (UNIFAP), Rod. Juscelino Kubitscheck, Km 02, 68902-280, Macapá, AP, Brasil

3. Coordenação de Biodiversidade, Instituto Nacional de Pesquisas da Amazônia (INPA), Caixa Postal 478, 69060-001, Manaus, AM, Brasil. (dnorris75@gmail.com)

4. Instituto Pró-Carnívoros, Caixa Postal 10, 12940-970, Atibaia, SP, Brasil. (fmichalski@procarnivoros.org.br, autor para contato)
\end{abstract}

\begin{abstract}
Richness and composition of vertebrates in active and inactive latrines of Pteronura brasiliensis (Carnivora, Mustelidae) in the Eastern Amazon, Brazil. This study aimed to evaluate the richness and composition of the medium and large sized vertebrates in active and inactive latrines of Giant otters [Pteronura brasiliensis (Gmelin, 1788)] in a Sustainable Use conservation unit in the Eastern Brazilian Amazon. The study was performed in 45 latrines along $230 \mathrm{~km}$ of the Falsino and Araguari rivers $\left(0^{\circ} 55^{\prime} \mathrm{N}, 51^{\circ} 35^{\prime} \mathrm{W}\right)$ and from this total, 24 presented fresh feces of Giant otters while 21 presented only old feces. From July to November 2012 each latrine was continuously monitored with a camera trap set to operate for 24 hours. The effort resulted in 458.8 camera trap/days, with 247.5 camera trap/days in latrines with fresh feces and 211.3 camera trap/ days with old feces. From this effort we obtained photos from a total of 22 vertebrate species. Most species photographed at latrines were mammals $(\mathrm{n}=13)$, followed by birds $(\mathrm{n}=6)$ and reptiles $(\mathrm{n}=3)$. The most frequently photographed species were paca [Cuniculus paca (Linnaeus, 1766), $\mathrm{n}$ $=21$ ], ocelot [Leopardus pardalis (Linnaeus, 1758), $\mathrm{n}=11$ ], white-tipped dove (Leptotila verreauxi Bonaparte, 1855, $\mathrm{n}=8$ ), giant otter [Pteronura brasiliensis (Gmelin, 1788), $\mathrm{n}=7$ ], and tapir [Tapirus terrestris (Linnaeus, 1758), $\mathrm{n}=6$ ], that accounted for 55.8\% of all records. Most records $(69.5 \%)$ were obtained in latrines with fresh feces and the number of vertebrate species was greater $(n=19)$ than in latrines with old feces $(n=15)$. However, the dissimilarity between the vertebrate communities of latrines with fresh and old feces did not differ statistically. However, the mean visitation of vertebrates to latrines with fresh feces was slightly higher than with old feces, although this difference was only marginally significant. There was an increase in records of felids [Leopardus pardalis, Leopardus wiedii (Schinz, 1821), and Panthera onca (Linnaeus, 1758)] in latrines with old feces, but it was only marginally significant. Thus, the presence of fresh feces of giant otters seems to increase the records of vertebrates, being especially important for groups with similar trophic guild.
\end{abstract}

KEYWORDS. Camera trap, interspecific interactions, food resource.

RESUMO. O estudo objetivou avaliar a riqueza e composição de vertebrados de médio e grande porte em latrinas ativas e inativas de ariranhas [Pteronura brasiliensis (Gmelin, 1788)], em uma Unidade de Conservação de Uso Sustentável na Amazônia Oriental Brasileira. O estudo foi realizado em 45 latrinas ao longo de $230 \mathrm{~km}$ nos rios Falsino e Araguari $\left(0^{\circ} 55^{\prime} \mathrm{N}, 51^{\circ} 35^{\prime} \mathrm{W}\right)$, sendo que desse total, 24 apresentaram fezes frescas e 21 fezes velhas de ariranhas. De julho a novembro de 2012, cada latrina foi monitorada com uma armadilha fotográfica programada para operar por 24 horas. O esforço de campo resultou em 458,8 armadilhas/dia, sendo 247,5 armadilhas/dia em latrinas com fezes frescas e 211,3 armadilhas/ dia com fezes velhas. Foram obtidos registros de 22 espécies de vertebrados. A maior parte das espécies registradas foram mamíferos $(\mathrm{n}=13)$, seguida por aves $(\mathrm{n}=6)$, e répteis $(\mathrm{n}=3)$. As espécies mais frequentemente fotografadas foram paca [Cuniculus paca (Linnaeus, 1766); $\mathrm{n}=21$ ], jaguatirica [Leopardus pardalis (Linnaeus, 1758); $\mathrm{n}=11$ ], juriti-pupu (Leptotila verreauxi Bonaparte, 1855; $\mathrm{n}=8$ ), ariranha [Pteronura brasiliensis (Gmelin, 1788); $\mathrm{n}=7$ ], e anta [Tapirus terrestris (Linnaeus, 1758); $\mathrm{n}=6$ ], que foram responsáveis por 55,8\% de todos os registros. A maior parte dos registros $(69,5 \%)$ foram obtidos em latrinas com fezes frescas e o número de espécies foi maior $(\mathrm{n}=19)$ do que os registrados em latrinas com fezes velhas $(\mathrm{n}=15)$. No entanto, a dissimilaridade entre a comunidade de vertebrados entre latrinas com fezes frescas e velhas não diferiu. A média de visitação em latrinas com fezes frescas foi ligeiramente superior do que em latrinas com fezes velhas, embora essa diferença tenha sido apenas marginalmente significativa. Entretanto, houve uma diminuição no número de registros de felinos [Leopardus pardalis, Leopardus wiedii (Schinz, 1821) e Panthera onca (Linnaeus, 1758)], marginalmente significativo em latrinas com fezes frescas. Dessa forma, a presença de fezes frescas em latrinas ativas de ariranhas parecem aumentar o registro de espécies de vertebrados, sendo especialmente importante para os grupos que apresentam guilda trófica similar.

PALAVRAS-CHAVE. Armadilha-fotográfica, interações interespecíficas, recurso alimentar.

Diversos fatores afetam a distribuição e abundância de vertebrados neotropicais. Tais fatores são compostos por um número de mecanismos de regulação que incluem desde predação natural até a exploração direta pelo homem (Peres, 1990; Bodmer, 1995; Peres \& Palacios, 2007). Para espécies territoriais vários fatores podem influenciar nas interações e alguns são determinantes na escolha e permanência no hábitat, como a disponibilidade de recursos, proteção contra predação e interações intra e interespecíficas com outras espécies (Hughes et al., 1994; DAVIDSON et al., 2012).
O principal modo de marcação de território é através de marcação odorífera ou deposição de fezes e urina, e representa um importante papel nas interações de espécies como ariranhas (Duplaix, 1980; Leuchtenberger \& MourÃo, 2009). A ariranha [Pteronura brasiliensis (Gmelin, 1788)] é um carnívoro social, endêmico da América do Sul e o maior representante da família Mustelidae (Duplaix, 1980; Eisenberg, 1989; CARTER \& Rosas, 1997; Rosas et al., 2008, 2009; Uscamaita \& BoDmer, 2009). Cada grupo de ariranhas possui seu território, que é defendido através de marcação e vocalizações, bem como por uma defesa 
ativa contra predadores e invasores conspecíficos (DUPLAIX, 1980; CARTER \& Rosas, 1997; Ribas \& Mourão 2004). A marcação do território é feita através de latrinas ("communal latrines") e estações de cheiro ("scent stations") (DuPLAIX, 1980; CARTer \& Rosas, 1997; Leuchtenberger \& Mourão, 2009). As latrinas são locais onde membros do grupo defecam e urinam (DuPLAIX, 1980; CARTER \& RosAs, 1997; Rosas et al., 2007; LeuChtENBERGER \& MourÃo, 2009) e são caracterizadas pela presença de remanescentes de escamas e ossos de peixes (GROENENDIJK et al., 2005). Ariranhas constroem latrinas em margens de rios, áreas abertas de fácil acesso a áreas florestais, regiões alagadiças ou igarapés, próximos a grandes rios e com pouca ou nenhuma vegetação de cobertura (Duplaix, 1980; Groenendijk et al., 2005). A frequência de uso das latrinas varia de acordo com o nível dos rios e sua localização, sendo que algumas podem ser ativas por longos períodos, enquanto outras são abandonadas, tornando-se inativas (DuPLAIX, 1980; ROSAS et al., 2007; LeuCHTENBERGER \& MourÃo, 2009).

Fezes de ariranhas podem representar uma fonte de recurso alimentar para algumas espécies de vertebrados (Leuchtenberger et al., 2012). Por serem depositadas em áreas abertas, latrinas ativas poderiam atrair vertebrados em busca de recursos (interação positiva), mas também ocasionar interações agonísticas (interação negativa) através de fatores diretos, como a presença de grupos de ariranha e/ou indiretos como o cheiro das fezes e urina, que poderia ser evitado por algumas espécies. Assim, esperase que a riqueza e composição de vertebrados de médio e grande porte seja maior em latrinas ativas de ariranhas.

O conhecimento básico sobre ariranhas, principalmente na Amazônia brasileira, ainda é bastante escasso (ICMBio, 2010; Lima et al., 2012). Estabelecer relações da ocorrência de ariranhas com outros vertebrados de médio e grande porte é um importante componente para o entendimento da espécie e de suas relações ecológicas. Neste estudo objetiva-se avaliar as diferenças na fauna de vertebrados de médio e grande porte em latrinas ativas e inativas de ariranhas em florestas tropicais. Para isso, foram testadas as seguintes hipóteses: (a) a riqueza e composição da comunidade de vertebrados de médio e grande porte é maior em latrinas ativas do que em latrinas inativas, e (b) os registros de espécies com guildas troficas semelhantes à da ariranha vão aumentar nas latrinas ativas.

\section{MATERIAL E MÉTODOS}

De julho a novembro de 2012 foram monitoradas 45 latrinas ao longo de $230 \mathrm{~km}$ nos rios Falsino e Araguari $\left(0^{\circ} 55^{\prime} \mathrm{N}, 51^{\circ} 35^{\prime} \mathrm{W}\right.$, Fig. 1), no entorno da Floresta Nacional do Amapá (FLONA/AP), Amazônia Oriental. Foram consideradas latrinas de ariranhas áreas limpas (sem presença de folhiço e com pouca vegetação rasteira) e com presença de fezes de ariranhas (Duplaix, 1980). Para maximizar a independência espacial entre as latrinas monitoradas, foi estabelecida uma distância linear mínima de $0,1 \mathrm{~km}$ (média $\pm \mathrm{DP}=27,5 \mathrm{~km} \pm 17,7$, mínimo-máximo $=0,15-67,5 \mathrm{~km}, \mathrm{n}=990$ pares de combinação) de acordo com LeuchtenBERGER et al. (2012).

Em cada latrina a condição das amostras de fezes de ariranhas (frescas ou velhas) foi classificada seguindo MichalsKi et al. (2011) utilizando as características de odor e umidade. Foram consideradas fezes frescas as que ainda possuíam odor muito forte (escalas de odor de 1 a 2) e umidade do conteúdo caracterizada como úmida por fora e por dentro (escala de umidade $=1$ ).



Fig. 1. Localização da área de estudo na Floresta Nacional do Amapá (FLONA/AP), Amazônia Oriental, Brasil, com as 45 latrinas de ariranhas (Pteronura brasiliensis) monitoradas (círculos cinza) entre julho e novembro de 2012. O polígono preto mostra os limites da FLONA/AP e o centro dos rios Falsino e Araguari. 
Cada latrina foi monitorada por uma armadilha fotográfica (Bushnell Trophy Cam, 8MP, Overland Park, KS, USA ou Tigrinus, modelo tradicional, Santa Catarina, Brasil), instalada a aproximadamente $30 \mathrm{~cm}$ de altura, voltada para o centro da latrina. As armadilhas foram programadas para operar continuamente (ou seja, 24 horas diariamente), com intervalo de $30 \mathrm{~s}$ entre fotos, durante um período médio de 10,2 dias (mínimo-máximo $=4-22$ dias, $\mathrm{DP}=3,3$ ). Para aumentar a independência entre registros fotográficos, fotos da mesma espécie na mesma armadilha fotográfica foram consideradas nas análises apenas quando o intervalo entre as mesmas for de pelo menos 30 minutos (LeUCHTENBERGER et al., 2012). As fotos obtidas de ariranhas em latrinas monitoradas foram excluídas das análises, pois não foram o objeto de interesse das questões a serem respondidas nesse estudo.

As espécies registradas nas armadilhas fotográficas foram identificadas com o auxílio de bibliografia de mamíferos (EMmons \& Ferr, 1997; Reis, 2011), aves (Sick, 1997; Sigrist, 2009) e répteis (VitT et al., 2008). As guildas tróficas foram determinadas de acordo com bases bibliográficas (Sick, 1997; VITT et al., 2008; SigRIST, 2009; ReIs et al., 2011).

O período de amostragem (julho a novembro de 2012) correspondeu à época de seca. Mesmo durante a seca houve dias com chuvas que poderiam afetar tanto o comportamento e atividade de espécies de vertebrados quanto à intensidade e persistência de fezes (e cheiro). Para testar se as chuvas amenas durante esse período afetaram o número de espécies registradas, os dados pluviométricos da área de estudo durante o período amostral foram obtidos da estação meteorológica mais próxima da área (Estação Coaracy Nunes - ANA, 2012). Sendo que a estação está localizada $<30 \mathrm{~km}$ das latrinas, assumimos que os valores diários refletem a chuva (em termos relativamente proporcionais) nas latrinas amostradas durante os períodos de amostragem. Para examinar se a riqueza de espécies registradas nas latrinas foi correlacionada com a média de chuva durante o período de amostragem, foi utilizado o teste de Spearman. A comparação da média de chuva e a condição da latrina com fezes frescas ou velhas foi analisada através do teste de Mann-Whitney.

A dissimilaridade entre a assembleia de vertebrados que foi registrada em cada latrina foi calculada com a distância Euclidiana. Para examinar se a composição de espécies registradas nas latrinas foi correlacionada com a distância Euclidiana entre latrinas, foi aplicado o teste de Mantel, com 1000 permutações. Foi utilizada uma análise de similaridade (ANOSIM), com 1000 permutações (CLARKE, 1993), para comparar estatisticamente a dissimilaridade entre as comunidades de vertebrados observadas em latrinas de ariranhas ativas (com amostras de fezes de ariranhas frescas) e inativas (apenas com amostras de fezes de ariranhas velhas). Foi avaliada a proporção de registros de cada espécie de vertebrados em latrinas ativas e inativas, que apresentaram pelo menos quatro registros em cada latrina, através do uso da função "prop.test" no Programa R
(R Development Core Team, 2012), que testa se a proporção em vários grupos é a mesma. A comparação da taxa de visitação de vertebrados em latrinas (número de fotos independentes por armadilhas-dia) e a condição da latrina (com fezes frescas ou velhas) foi analisada através de teste de Mann-Whitney. Para avaliar a diferença de vertebrados carnívoros e de felinos em latrinas ativas e inativas foi utilizado o teste Qui-quadrado. Todas as análises foram realizadas com o Programa R versão 2.15.1 (R DEVELOPMENT Core Team, 2012), utilizando o pacote "vegan".

\section{RESULTADOS}

Do total de latrinas monitoradas no período, 24 apresentaram fezes frescas de ariranhas (ativas) enquanto as outras 21 exibiram apenas fezes velhas de ariranhas (inativas). No total, o esforço de campo resultou em 458,8 armadilhas/dia, sendo 247,5 armadilhas/dia em latrinas ativas e 211,3 armadilhas/dia em latrinas inativas.

A média de pluviosidade na região foi de $0,29 \mathrm{~mm}$ para todas as 45 latrinas monitoradas, variando de 0 a 20 mm (Tab. I). Os dados pluviométricos da área de estudo não foram correlacionados com o número de espécies registradas durante o período amostral (Correlação de Spearman, $\left.\mathrm{r}_{\mathrm{s}}=0,133, P=0,47\right)$ e não existe uma diferença significativa entre a pluviosidade média durante os períodos de amostragem das latrinas ativas e inativas (Teste de Mann-Whitney, $\mathrm{W}=122, P=0,7336$ ).

Foram obtidos registros de 22 espécies de vertebrados (Tab. II) nas latrinas de ariranhas monitoradas na área de estudo. A composição de espécies não foi correlacionada com a distância entre latrinas (Teste de Mantel, $P<0,120$ ). A maioria das espécies registradas foram mamíferos $(\mathrm{n}=$ $13)$, seguida por aves $(n=6)$ e répteis $(n=3)$. As espécies mais frequentemente fotografadas foram paca [Cuniculus paca (Linnaeus, 1766), $\mathrm{n}=21$ ], jaguatirica [Leopardus pardalis (Linnaeus, 1758), $\mathrm{n}=11$ ], juriti-pupu (Leptotila verreauxi Bonaparte, 1855; $\mathrm{n}=8$ ), ariranha (Pteronura brasiliensis; $\mathrm{n}=7$ ), e anta [Tapirus terrestris (Linnaeus, 1758); $\mathrm{n}=6$ ], que foram responsáveis por $55,8 \%$ de todos os registros fotográficos obtidos.

Somente as proporções de registros de paca $(C$. paca) apresentaram diferenças significativas entre as latrinas ativas e inativas de ariranhas monitoradas (Tab. II).

Do total de registros fotográficos de vertebrados obtidos em latrinas, 69,5\% foram em latrinas ativas de ariranhas (Fig. 2). Da mesma forma, o número de espécies registrados em latrinas ativas foi maior $(n=19)$ do que os registrados em latrinas inativas $(\mathrm{n}=15)$. No entanto, a dissimilaridade entre a comunidade de vertebrados entre latrinas ativas e inativas não diferiu significativamente ( $R=-0,048, P=0,742)$. Também não houve diferença significativa entre as espécies de vertebrados carnívoras em latrinas ativas e inativas $\left(\chi^{2}=0,474 ; P=0,491\right)$, porém houve diferença marginalmente significativa na presença de felinos em latrinas inativas $(76,9 \%)\left(\chi^{2}=3,769 ; P=0,052\right)$.

A média de visitação (número de fotos independentes 
Tab. I. Identificação da latrina (ID), localização e dados pluviométricos (média, desvio padrão, e mínima-máxima) do período de amostragem das latrinas de ariranhas (Pteronura brasiliensis) monitoradas com armadilhas fotográficas. Dados pluviométricos obtidos da Estação Coaracy-Nunes, Amapá (ANA, 2012).

\begin{tabular}{|c|c|c|c|c|c|c|}
\hline ID & Zona & Latitude & Longitude & Média $(\mathrm{mm})$ & Desvio padrão & Mín-Máx (mm) \\
\hline 1 & $22 \mathrm{~N}$ & 413926 & 110147 & 3,8 & 6 & $0-20$ \\
\hline 2 & $22 \mathrm{~N}$ & 411241 & 109847 & 0,33 & 0,52 & $0-1$ \\
\hline 3 & $22 \mathrm{~N}$ & 415777 & 108304 & 0,25 & 0,5 & $0-1$ \\
\hline 4 & $22 \mathrm{~N}$ & 451599 & 131234 & 0,4 & 0,89 & $0-2$ \\
\hline 5 & $22 \mathrm{~N}$ & 444289 & 120228 & 0,4 & 0,89 & $0-2$ \\
\hline 6 & $22 \mathrm{~N}$ & 421501 & 109218 & 0,66 & 2 & $0-6$ \\
\hline 7 & $22 \mathrm{~N}$ & 417525 & 108105 & 0,66 & 2 & $0-6$ \\
\hline 8 & $22 \mathrm{~N}$ & 421241 & 109387 & 0,66 & 2 & $0-6$ \\
\hline 9 & $22 \mathrm{~N}$ & 438622 & 115822 & 0,66 & 2 & $0-6$ \\
\hline 10 & $22 \mathrm{~N}$ & 441340 & 115315 & 0,66 & 2 & $0-6$ \\
\hline 11 & $22 \mathrm{~N}$ & 442111 & 116092 & 0,66 & 2 & $0-6$ \\
\hline 12 & $22 \mathrm{~N}$ & 445512 & 120014 & 0,75 & 2,12 & $0-6$ \\
\hline 13 & $22 \mathrm{~N}$ & 447059 & 121324 & 0,75 & 2,12 & $0-6$ \\
\hline 14 & $22 \mathrm{~N}$ & 425293 & 110300 & 0 & 0 & 0 \\
\hline 15 & $22 \mathrm{~N}$ & 422686 & 112722 & 0 & 0 & 0 \\
\hline 16 & $22 \mathrm{~N}$ & 416665 & 108141 & 0 & 0 & 0 \\
\hline 17 & $22 \mathrm{~N}$ & 415837 & 107436 & 0 & 0 & 0 \\
\hline 18 & $22 \mathrm{~N}$ & 409341 & 108117 & 0 & 0 & 0 \\
\hline 19 & $22 \mathrm{~N}$ & 415766 & 109517 & 0 & 0 & 0 \\
\hline 20 & $22 \mathrm{~N}$ & 441094 & 115201 & 0 & 0 & 0 \\
\hline 21 & $22 \mathrm{~N}$ & 441245 & 115199 & 0 & 0 & 0 \\
\hline 22 & $22 \mathrm{~N}$ & 441663 & 117478 & 0 & 0 & 0 \\
\hline 23 & $22 \mathrm{~N}$ & 448825 & 122907 & 0 & 0 & 0 \\
\hline 24 & $22 \mathrm{~N}$ & 450550 & 129041 & 0 & 0 & 0 \\
\hline 25 & $22 \mathrm{~N}$ & 450531 & 128028 & 0 & 0 & 0 \\
\hline 26 & $22 \mathrm{~N}$ & 449433 & 122835 & 0 & 0 & 0 \\
\hline 27 & $22 \mathrm{~N}$ & 447694 & 122236 & 0 & 0 & 0 \\
\hline 28 & $22 \mathrm{~N}$ & 411060 & 108173 & 0,5 & 1,73 & $0-6$ \\
\hline 29 & $22 \mathrm{~N}$ & 450516 & 130017 & 0,46 & 1,66 & $0-6$ \\
\hline 30 & $22 \mathrm{~N}$ & 450438 & 126201 & 0,46 & 1,66 & $0-6$ \\
\hline 31 & $22 \mathrm{~N}$ & 443653 & 117871 & 0,5 & 1,73 & $0-6$ \\
\hline 32 & $22 \mathrm{~N}$ & 433851 & 112562 & 0,5 & 1,73 & $0-6$ \\
\hline 33 & $22 \mathrm{~N}$ & 451563 & 132979 & 0 & 0 & 0 \\
\hline 34 & $22 \mathrm{~N}$ & 450627 & 137894 & 0 & 0 & 0 \\
\hline 35 & $22 \mathrm{~N}$ & 455265 & 140784 & 0 & 0 & 0 \\
\hline 36 & $22 \mathrm{~N}$ & 452381 & 139957 & 0 & 0 & 0 \\
\hline 37 & $22 \mathrm{~N}$ & 452036 & 139088 & 0 & 0 & 0 \\
\hline 38 & $22 \mathrm{~N}$ & 450952 & 138700 & 0 & 0 & 0 \\
\hline 39 & $22 \mathrm{~N}$ & 390262 & 135128 & 0 & 0 & 0 \\
\hline 40 & $22 \mathrm{~N}$ & 388528 & 130373 & 0 & 0 & 0 \\
\hline 41 & $22 \mathrm{~N}$ & 390513 & 123694 & 0 & 0 & 0 \\
\hline 42 & $22 \mathrm{~N}$ & 394316 & 117106 & 0 & 0 & 0 \\
\hline 43 & $22 \mathrm{~N}$ & 398771 & 118212 & 0 & 0 & 0 \\
\hline 44 & $22 \mathrm{~N}$ & 400580 & 115426 & 0 & 0 & 0 \\
\hline 45 & $22 \mathrm{~N}$ & 405360 & 113768 & 0 & 0 & 0 \\
\hline
\end{tabular}

de vertebrados) em latrinas ativas foi ligeiramente superior $(0,260$ fotos/armadilhas-dia, min-máx $=0-0,761)$ do que em latrinas inativas $(0,141$ fotos/armadilhas-dia, min-máx $=0-0,570$, Fig. 2). No entanto, essa diferença foi apenas marginalmente significativa (Teste de Mann-Whitney, W $=179,5, P=0,096$ ).

\section{DISCUSSÃO}

Os registros de vertebrados obtidos nas latrinas de ariranhas demonstraram que (1) a riqueza e composição de vertebrados de médio e grande porte na área de estudo não diferiu entre latrinas ativas e inativas, (2) apenas a 
Tab. II. Espécies de vertebrados registradas com armadilhas fotográficas em latrinas de ariranhas (Pteronura brasiliensis) na Floresta Nacional do Amapá entre julho e novembro de 2012 (FRU, frugívoros; HER, herbívoros; DET, detritívoros; INS, insetívoros; CAR, carnívoros; PIS, piscívoros; OMN, onívoros) ( a , bibliografia utilizada para determinar a guilda trófica: ${ }^{1}$ ReIS, 2011; ${ }^{2}$ SicK, 1997; ${ }^{3}$ SigRIST, 2009; ${ }^{4}$ VITT et al., 2008 ) ( ${ }^{b}$, teste de proporção entre registros em latrinas com fezes frescas e velhas: ${ }^{+}$não significativo, $\left.{ }^{*} P<0,05\right)$.

\begin{tabular}{|c|c|c|c|c|}
\hline \multirow[b]{2}{*}{ Espécie $^{\mathrm{a}}$} & \multirow[b]{2}{*}{$\begin{array}{l}\text { Guilda } \\
\text { trófica }\end{array}$} & \multicolumn{2}{|c|}{$\begin{array}{l}\text { Registros nas armadilhas } \\
\text { fotográficas }\end{array}$} & \multirow[b]{2}{*}{ Total $^{\mathrm{b}}$} \\
\hline & & $\begin{array}{c}\text { Latrinas } \\
\text { ativas }\end{array}$ & $\begin{array}{l}\text { Latrinas } \\
\text { inativas }\end{array}$ & \\
\hline \multicolumn{5}{|l|}{ Répteis } \\
\hline Ameiva ameiva (Linnaeus, 1758) ${ }^{4}$ & INS & 1 & 0 & 1 \\
\hline Iguana iguana (Linnaeus, 1758) ${ }^{4}$ & HER & 1 & 3 & $4^{+}$ \\
\hline Kentropyx calcarata $($ Spix, 1825) & INS & 3 & 0 & 3 \\
\hline \multicolumn{5}{|l|}{ Aves } \\
\hline Botaurus pinnatus (Wagler, 1829) (2,3 $^{2,3}$ & $\mathrm{OMN}$ & 1 & 0 & 1 \\
\hline Urubitinga urubitinga (Gmelin, 1788) & CAR & 3 & 1 & $4^{+}$ \\
\hline Crax alector (Linnaeus, 1766) & $\mathrm{OMN}$ & 3 & 1 & $4^{+}$ \\
\hline Crypturellus cinereus (Gmelin, 1789) & $\mathrm{OMN}$ & 1 & 0 & 1 \\
\hline Leptotila verreauxi (Bonaparte, 1855$)^{2,3}$ & FRU & 6 & 2 & $8^{+}$ \\
\hline Mesembrinibis cayennensis (Gmelin, 1789) & OMN & 3 & 1 & $4^{+}$ \\
\hline \multicolumn{5}{|l|}{ Mamíferos } \\
\hline Cuniculus paca (Linnaeus, 1766) ${ }^{1}$ & FRU & 16 & 5 & $21^{*}$ \\
\hline Dasyprocta agouti (Illiger, 1811) ${ }^{1}$ & FRU & 4 & 1 & $5^{+}$ \\
\hline Didelphis marsupialis (Linnaeus, 1758) ${ }^{1}$ & $\mathrm{OMN}$ & 0 & 1 & 1 \\
\hline Hydrochoerus hydrochaeris (Linnaeus, 1766) ${ }^{1}$ & HER & 1 & 1 & 2 \\
\hline Leopardus pardalis (Linnaeus, 1758) ${ }^{1}$ & CAR & 3 & 8 & $11^{+}$ \\
\hline Leopardus wiedii $(\mathrm{Schinz}, 1821)^{1}$ & CAR & 0 & 1 & 1 \\
\hline Lontra longicaudis (Olfers, 1818) ${ }^{1}$ & $\mathrm{CAR} / \mathrm{PIS}$ & 2 & 0 & 2 \\
\hline Marmosa sp. ${ }^{1}$ & INS/OMN & 3 & 0 & 3 \\
\hline Mazama americana (Erxleben, 1777) ${ }^{1}$ & HER & 2 & 1 & 3 \\
\hline Panthera onca (Linnaeus, 1758) ${ }^{1}$ & CAR & 0 & 1 & 1 \\
\hline Pteronura brasiliensis (Gmelin, 1788) ${ }^{1}$ & CAR/PIS & 7 & 0 & 7 \\
\hline Pequeno roedor (não identificado) & - & 1 & 1 & 2 \\
\hline Tapirus terrestris (Linnaeus, 1758) ${ }^{1}$ & HER & 5 & 1 & $6^{+}$ \\
\hline Total de registros obtidos & & 66 & 29 & 95 \\
\hline Esforço (armadilhas/dia) & & 247,5 & 211,3 & 458,8 \\
\hline Latrinas monitoradas & & 24 & 21 & 45 \\
\hline
\end{tabular}

proporção dos registros de paca (C. paca) demonstrou diferença significativa entre as latrinas ativas e inativas, (3) embora haja uma tendência a ocorrer maior registro de vertebrados em latrinas ativas, esse aumento não foi significativo, e (4) espécies com guilda trófica similar ao das ariranhas, como os carnívoros registrados nas latrinas, não diferiram em relação a latrinas ativas e inativas; no entanto, felinos tenderam a utilizar mais frequentemente as áreas com menor frequência de ariranhas (latrinas inativas), mas essa tendência foi apenas marginalmente significativa.

Nossos resultados corroboram aos encontrados por LeuChteNBERGer et al. (2012) no Pantanal, que também não constataram diferença na taxa de visitação e na composição de vertebrados em latrinas ativas e inativas. A presença da comunidade de vertebrados registrados pode estar relacionada a características físicas das latrinas, e não exclusivamente com a idade das fezes. Além disso, espécies de vertebrados que utilizam preferencialmente ambientes próximos de corpos d'água tendem a utilizar essas áreas com maior frequência (EMmons \& FERR, 1997; SicK, 1997; VitT et al., 2008; Sigrist, 2009; ReIs et al., 2011).

Dentre as espécies de vertebrados, a paca (Cuniculus paca) apresentou maior número de registros em latrinas ativas $(76,19 \%, P<0.05)$. A paca é uma espécie frugívora que ocorre preferencialmente em florestas tropicais úmidas, próximo a cursos de água (Zucaratto et al., 2010; Reis, 2011), dorme em tocas presentes em barrancos (ReIs et al., 2011) e utiliza trilhas próprias para os locais de alimentação (Perez, 1992). Dessa forma, o maior número de registros dessa espécie em latrinas pode estar relacionado com as áreas de uso em comum, e não necessariamente com a frequência de fezes frescas de ariranhas.

O registro de vertebrados carnívoros não foi diferente em latrinas ativas e inativas, porém um maior número de registros de espécies de felinos registrados como a onça pintada [Panthera onca (Linnaeus, 1758)], jaguatirica [Leopardus pardalis (Linnaeus, 1758)] e gato maracajá [Leopardus wiedii (Schinz, 1821)] em latrinas 


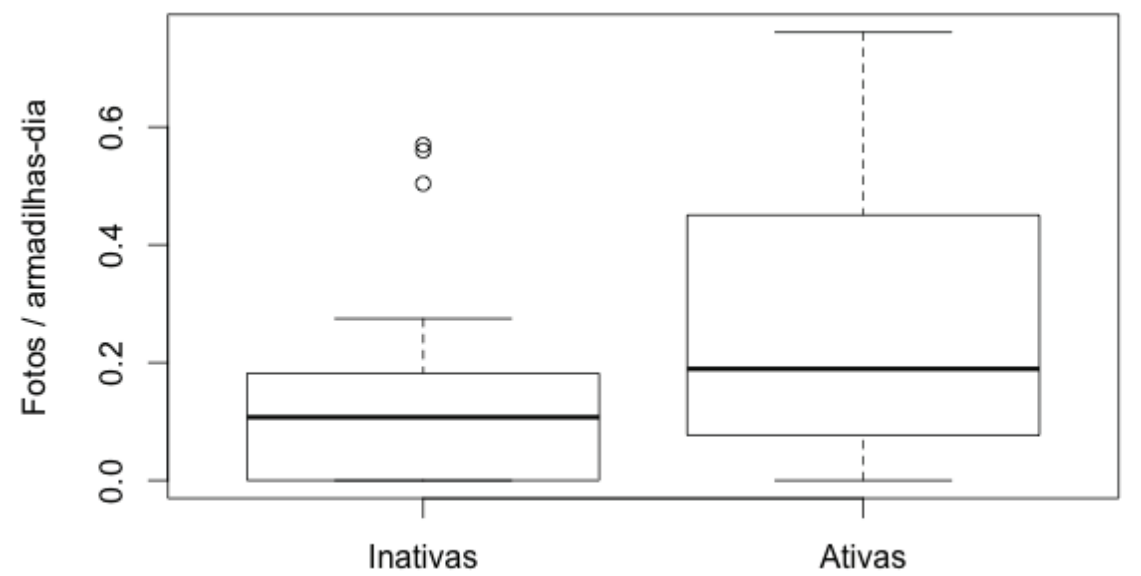

Fig. 2. Número de fotos independentes por armadilhas-dia em latrinas ativas e inativas de ariranhas monitoradas entre julho e novembro de 2012 na Floresta Nacional do Amapá (FLONA/AP), Amazônia Oriental, Brasil. A linha preta mostra a mediana do número de fotos independentes/armadilhas-dia.

inativas, poderia indicar que estes predadores evitam encontros com ariranhas.

Dentre as aves registradas, o gavião preto [(Urubitinga urubitinga (Gmelin, 1788)] foi a única ave carnívora e, embora a diferença entre as proporções de registros em latrinas ativas e inativas não tenha sido estatisticamente diferente, o maior número de registros ocorreu em latrinas ativas. Essa espécie comumente habita margens de rios (SIGRIST, 2009) e sua guilda trófica poderia indicar um possível aproveitamento de recurso alimentar em latrinas como observado por LeuchtenBerger et al. (2012) para Ortalis canicollis, Caracara plancus, Aramides cajanea e Iguana iguana. Da mesma forma, observamos um maior número de registros de mutum [Crax alector (Linnaeus, 1766)] em latrinas ativas. Essa espécie habita matas de terra firme e matas ripárias ao norte do rio Amazonas e é onívora, forrageando principalmente no solo (Sick, 2009), onde as fezes de ariranhas poderiam ser encontradas. LEUCHTENBERGER et al. (2012) registraram coprofagia de fezes de ariranhas por mutum em latrinas, o que poderia indicar uma possibilidade de complementação da dieta, já que há registros de consumo de solo rico em sais pela espécie (Del Hoyo, 1994).

Entre os répteis, a iguana [Iguana iguana (Linnaeus, 1758)] foi a espécie com maior número de registros. A iguana é herbívora e as fêmeas realizam migrações para nidificar ao longo de margens de rios, córregos e riachos (CAmpos, 2004). Leuchtenberger et al. (2012) também constataram a ingestão de fezes de ariranha por iguanas, sugerindo que a coprofagia poderia auxiliar na obtenção de microorganismos que digerem alguns componentes químicos presentes em plantas, podendo também ser uma fonte extra de cálcio (CAMPOS et al., 2011). Embora os registros fotográficos obtidos na FLONA/AP não permitiram que fosse identificada claramente coprofagia pelos vertebrados, é possível que algumas espécies utilizem fezes de ariranhas para complementação da dieta.

Os estudos que avaliam o uso de latrinas normalmente são focados na dieta de ariranhas (RosAs et al., 1999; RiBAS, 2004) ou na atividade da espécie (UtrERAS \& Pinos, 2003; Leuchtenberger et al., 2014). Dessa forma, vários aspectos da interação entre populações de ariranhas e outras espécies de vertebrados de médio e grande porte em áreas de marcação de territórios, como as latrinas, ainda permanecem como uma lacuna de conhecimento.

Os resultados apresentados em nosso estudo demonstram que latrinas de ariranhas podem atrair vertebrados de médio e grande porte, seja como fonte de alimento ou pelo hábitat similar que ocupam.

Agradecimentos. Essa pesquisa recebeu apoio do Instituto Chico Mendes de Conservação da Biodiversidade (ICMBio) e do CNPq (Processo: 477629/2011-3). A Universidade Federal do Amapá forneceu apoio logístico. Agradecemos o apoio do IBAMA pela autorização de pesquisa (IBAMA/SISBIO n ${ }^{\circ}$ 26653-2). CMT recebeu bolsa de mestrado da CAPES e DN bolsa de pós-doutorado do CNPq. Alvino Pantoja,

"Cutião" e Isabel Oliveira auxiliaram nas atividades de campo.

\section{REFERÊNCIAS BIBLIOGRÁFICAS}

ANA. 2012. Hidroweb. Disponível em: <http://hidroweb.ana.gov.br>. Acesso em: 04.05.2013.

Bodmer, R. E. 1995. Managing Amazonian wildlife: Biological correlates of game choice by detribalized hunters. Ecological Applications 5(4):872-877.

Campos, Z. 2004. Estudo das iguanas no Pantanal. Embrapa Pantanal 67(1):1-2.

Campos, Z.; Leuchtenberger, C.; Desbiez, A. L. J. \& Mourão, G. 2011. Iguana iguana (Green iguana). Coprophagy. Herpetological Review 42(4):604-605.

CARTer, S. K. \& Rosas, F. C. W. 1997. Biology and conservation of the giant otter Pteronura brasiliensis. Mammal Review 27(1):1-26.

Clarke, K. R. 1993. Non-parametric multivariate analysis of changes in community structure. Australian Journal of Ecology 18(1):117-143.

Davidson, Z.; Valeix, M.; Loveridge, A.; Hunt, J. E.; Johnson, P. J.; Madzikanda, H. \& MacDonald, D. W. 2012. Environmental determinants of habitat and kill site selection in a large carnivore: scale matters. Journal of Mammalogy 93(3):677-685.

Del Royo, J. 1994. Family Cracidae. New world vultures to guineafowl. In: Del Hoyo, J. et al. eds. Handbook of the Birds of the World, Vol. 2. Barcelona, Lynx Edic., p. 310-363.

DuplaIX, N. 1980. Observations on the ecology and behavior of the giant river otter Pteronura brasiliensis in Suriname. Revue Ecologique (Terre Vie) 1(34):496-620.

Eisenberg, J. F. 1989. The Northern Neotropics: Panama, Colombia, 
Venezuela, Guyana, Suriname, French Guiana. In: Eisenberg, J. F. ed. Mammals of the Neotropics. Chicago, University of Chicago Press. 550p.

Emmons, L. H. \& FeER, F. 1997. Neotropical rainforest mammals: A field guide. Chicago, The University of Chicago Press. 307p.

Groenendijk, J.; Halej, F.; Duplaix, N.; Reuther, C.; van Damme, P.; Schenck, C.; Staib, E.; Wallace, R.; Wandemarin, H.; Notin, R.; Marmontel, M.; Rosas, F. C. W.; Mattos, G. E. DE; Evangelista, E.; Utreras, V.; Lasso, G.; Jacques, H.; Matos, K.; Roopsind, I. \& Botello, J. C. 2005. Surveying and monitoring distribution and population trends of the Giant otter (Pteronura brasiliensis): Guidelines for a Standardisation of Survey Methods as recommended by the Giant Otter Section of the IUCN/SSC Otter Specialist Group. Frankfurt, Frankfurt Zoological Society. 99p.

Hughes, J. J.; Ward, D. \& Perrin, M. R. 1994. Predation and competition affect habitat selection and activity in desert gerbils. Ecology 75(5):1397-1405.

ICMBıo. 2010. Sumário Executivo do Plano de Ação Nacional para a Conservação da Ariranha. Disponível em: $<$ http://www.icmbio. gov.br/portal/images/stories/docs-plano-de-acao/pan-ariranha/ sumario ariranhas lontras.pdf $>$. Acesso em: 12.10.2012.

Leuchtenberger, C. \& Mourão, G. 2009. Scent-Marking of Giant Otter in the Southern Pantanal, Brazil. Ethology 115(3):210-216.

Leuchtenberger, C.; Ribas, C.; Magnusson, W. \& Mourão, G. 2012. To each his own taste: latrines of the giant otter as a food resource for vertebrates in Southern Pantanal, Brazil. Studies on Neotropical Fauna and Environment 47(2):81-85.

Leuchtenberger, C.; Zucco, C. A.; Ribas, C.; Magnusson, W. \& Mourão, G. 2014. Activity patterns of giant otters recorded by telemetry and camera traps. Ethology Ecology \& Evolution 26(1):19-28.

Lima, D. S.; Marmontel, M. \& Bernard, E. 2012. Site and refuge use by giant river otters (Pteronura brasiliensis) in the Western Brazilian Amazonia. Journal of Natural History 46(11-12):729-739.

Michalski, F.; Valdez, F. P.; Norris, D.; Zieminski, C.; Kashivakura, C. K.; Trinca, C.; Smith, H. B.; Vynne, C.; Wasser, S. K.; Metzger, J. P. \& EIZIRIK, E. 2011. Successful carnivore identification with faecal DNA across a fragmented Amazonian landscape. Molecular Ecology Resources 11(5):862-871.

Peres, C. A. 1990. Effects of hunting on western Amazonian primate communities. Biological Conservation 54(1):47-59.

Peres, C. \& Palacios, E. 2007. Basin-Wide Effects of Game Harvest on Vertebrate Population Densities in Amazonian Forests: Implications for Animal-Mediated Seed Dispersal. Biotropica 39(3):304-315.

Perez, E. M. 1992. Agouti paca. Mammalian Species 404(1):1-7.

R Development Core Team. 2012. R: a language and environment for statistical computing. R Foundation for Statistical Computing, Vienna, Austria. Disponível em: $<$ http://www.R-project.org $>$. Accesso em: 18.12.2012.

Reis, N. R.; Peracchi, A. L.; Pedro, W. A. \& Lima, I. P. de 2011. Mamíferos do Brasil. 2. Londrina: Nélio R dos Reis. 439p.

Ribas, C. \& Mourão, G. 2004. Intraspecific Agonism between Giant Otter Groups. IUCN Otter Specialist Group Bulletin 21(1):1-4.

Rosas, F. C. W.; Zuanon, J. A. S. \& Carter, S. K. 1999. Feeding ecology of the giant otter, Pteronura brasiliensis. Biotropica 31(3):502-506.

Rosas, F. C. W.; Mattos, G. L. De \& Cabral, M. M. M. 2007. The use of hydroelectric lakes by giant otters Pteronura brasiliensis: Balbina lake in central Amazonia, Brazil. Oryx 41(4):520-524.

Rosas, F. C. W.; Rocha, C. S. da; Mattos, G. E. de \& Lazzarini, S. M. 2009. Body weight-length relationships in giant otters (Pteronura brasiliensis) (Carnivora, Mustelidae). Arquivos Brasileiros de Biologia e Tecnologia 52(3):587-591.

Rosas, F. C. W.; Waldemarin, H. \& Mattos, G. E. de 2008. Ariranha, Pteronura brasiliensis (Zimmermann, 1780). In: MaCHADO, A. B. M.; Drummond, G. M. \& Paglia, A. P. eds. Livro Vermelho da Fauna Brasileira Ameaçada de Extinção. v.1. Belo Horizonte, Fundação Biodiversitas, p.800-801.

Sick, H. 1997. Ornitologia brasileira. Rio de Janeiro, Nova Fronteira. 902 p.

Sigrist, T. 2009. Guia de Campo Avis Brasilis - Avifauna Brasileira: Descrição das Espécies $=$ The Avis Brasilis Field Guide to the Birds of Brazil: Species Accounts. São Paulo, Avis Brasilis. 600p.

Uscamaita, M. R. \& Bodmer, R. 2009. Recovery of the endangered giant otter Pteronura brasiliensis on the Yavarí-Mirín and Yavarí Rivers: a sucess story for CITES. Oryx 44(1):83-88.

Utreras, V. B. \& Pinos, L. 2003. Camera trap use for studying giant otters (Pteronura brasiliensis) in the Yasuní Biosphere Reserve, Ecuadorian Amazon. IUCN Otter Specialist Group Bulletin 20(2):69-70.

Vitt, L.; Magnusson, W. E.; Pires, T. C. A. \& Lima, A. P. 2008. Guia de Lagartos da Reserva Adolpho Ducke, Amazônia Central = Guide to the Lizards of the Reserve Adolpho Ducke, Central Amazonia. Manaus, Atterna Design Editorial. 176p.

Zucaratto, R.; Carrara, R. \& Franco, B. K. S. 2010. Dieta de paca (Cuniculus paca) usando métodos indiretos numa área de cultura agrícola na Floresta Atlântica brasileira. Biotemas 23(1):235-239.

Recebido em 4 de novembro de 2013. Aceito em 28 de março de 2014. ISSN 0073-4721

Artigo disponível em: www.scielo.br/isz 